\title{
GW23-e1192 EFFECTS OF ATORVASTATIN ON METHYLATION AND MRNA EXPRESSION OF BCL-2 IN HYPERLIPIDAEMIA WISTAR RATS
}

doi:10.1136/heartjnl-2012-302920a.17

Wang Yiyong, Jia Shaobin. Center of Cardiology, General Hospital, Ningxia Medical University

Objectives To investigate effects of atorvastatin on methylation status of bcl-2 gene promoter and mRNA expression of bcl-2 in aortic tissue of hyperlipidaemia Wistar rats.

Methods 66 Wistar rats were equally randomised into three groups: control group, hyperlipidaemia group and hyperlipoidaemia rat with atorvastatin group. The rats in control group were fed a normal chaw, and the other groups were fed a chaw formula as designed for 12 weeks. From the 12th week gavage experiment, after 4 weeks of heart blood was then drawn for detection of serum cholesterol, triglyceride, low density lipoprotein-cholesterol, high density lipoprotein-cholesterol; aortic nucleoprotein was extracted for detection of DNA methyltransferase activity. The methylation specific polymerase chain reaction (MSP) method was used to detect bcl-2 gene methylation in aortic tissue of control group, hyperlipidemia group and hyperlipoidemia rat with atorvastatin group. The expression of bcl-2 mRNA in aortic tissue of control group and hyperlipidaemia group Wistar rats was detected by real-time quantitative polymerase chain reaction.

Results A high-fat diet for 12 weeks is sufficient to induce hyperlipidaemia; Atorvastatin supplementation to the rats fed the highfat diet prevented an elevation total cholesterol (TC), triglyceride (TG), low density lipoprotein-cholesterol (LDL-C) and increase high density lipoprotein-cholesterol (HDL-C) levels in the serum $(\mathrm{p}<0.05)$ and morphological changes in the thoracic aorta. Compared with the control group, hyperlipidaemia group significantly increased DNA methyltransferase activity and methylation status of $b c l-2$ gene promoter $(p<0.05)$, the expression of bcl-2 mRNA was decreased in hyperlipidaemia groups $(p<0.05)$. Compared with the hyperlipidaemia group, atorvastatin group significantly decreased DNA methyltransferase activity and methylation status of bcl-2 gene promoter $(p<0.05)$, the expression of bcl-2 mRNA was increased in hyperlipidaemia groups $(p<0.05)$. 
Conclusions Atorvastatin supplementation can blunt the rise in methylation status of bcl-2 gene promoter but also can increase the expression of bcl-2 mRNA in the aorta of rats with hyperlipidaemia. The study provide new ideas for prevention and delay of atherosclerosis. 